UCRL-JC-129854

PREPRINT

\title{
Beam Control and Laser Characterization for NIF
}

\author{
S. J. Boege \\ E. S. Bliss
}

This paper was prepared for submittal to the 1998 American Nuclear Society Annual Meeting

Nashville, TN

June 7-11, 1998

June 10, 1998

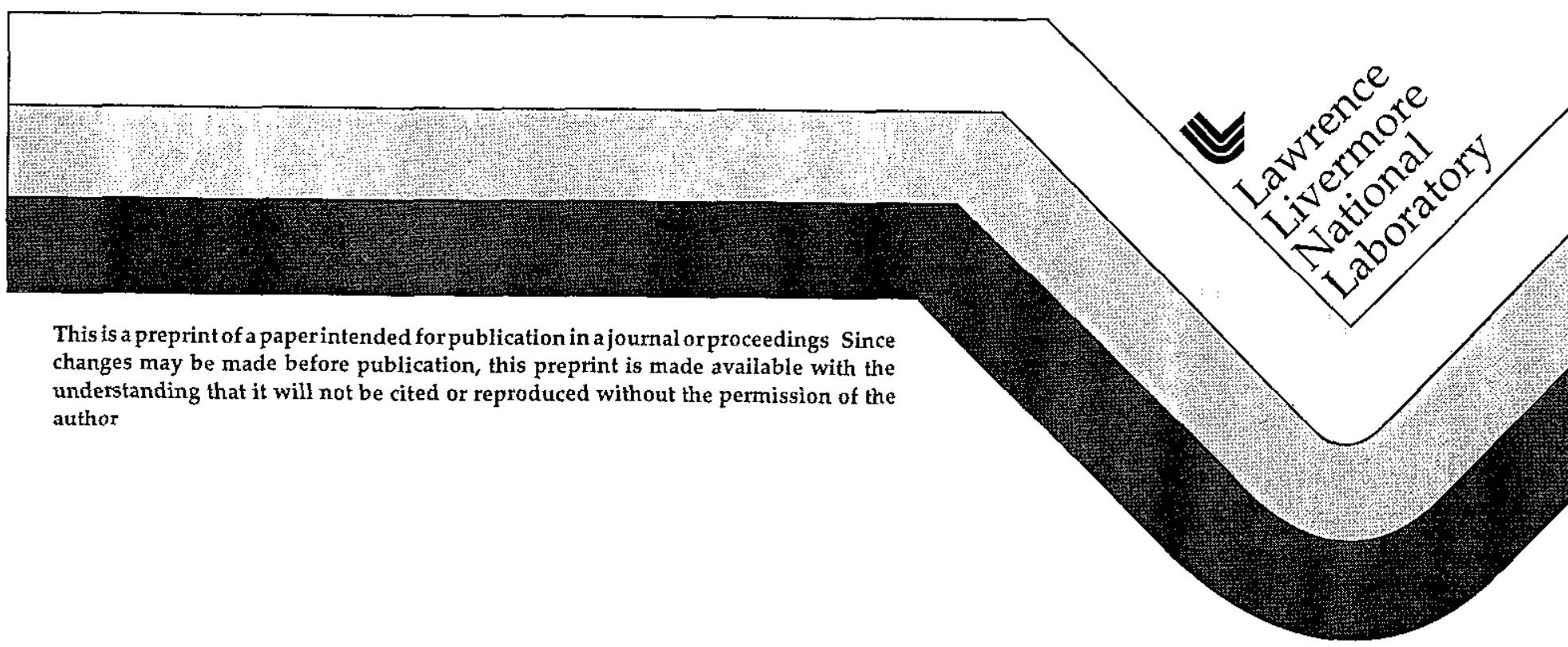




\section{DISCLAIMER}

This document was prepared as an account of work sponsored by an agency of the United States Government. Neither the United States Government nor the University of California nor any of their employees, makes any warranty, express or implied, or assumes any legal liability or responsibility for the accuracy, completeness, or usefulness of any information, apparatus, product, or process

disclosed, or represents that its use would not infringe privately owned rights. Reference herein to any specific commercial product, process, or service by trade name, trademark, manufacturer, or otherwise, does not necessarily constitute or imply its endorsement, recommendation, or favoring by the United States Government or the University of California. The views and opinions of authors expressed herein do not necessarily state or reflect those of the United States Government or the University of California, and shall not be used for advertising or product endorsement purposes. 
Steven J Boege

Lawrence Livermore National Laboratory

P O Box 808, L-495

Livermore, CA 94551

(925)423-6931
Erlan S Bliss

Lawrence Livermore National Laboratory

P O Box 808, L-495

Livermore, CA 94551

(925) $422-5483$

\section{ABSTRACT}

The demanding energy, power, pulse shape, focusability, pointing, and availability requirements placed on the 192 National Ignition Facility (NIF) beams lead to the need for an automatic operation capability that is well beyond that of previous inertial confinement fusion (ICF) lasers Alignment, diagnostic, and wavefront correction subsystems are integrated in an approach that, by permitting maximal sharing of instrumentation between subsystems, meets performance requirements at a reasonable cost

\section{INTRODUCTION}

NIF delivers light from 192 laser beams to a common target The system is designed to produce a $20 \mathrm{~ns}, 18 \mathrm{MJ}$ pulse of $351 \mathrm{~nm}$ light every 8 hours Each beam makes multiple passes through the amplifier chain Clear aperture, system maintenance, and target physics requirements necessitate active alignment and characterization of each laser chain, from the output of the regenerative amplifier to its target chamber (TC) aimpoint Target physics, specifically focal spot size, requirements also necessitate correction of transmitted wavefront Active wavefront correction is essential due to the dynamic nature of aberrations imparted on beams by thermally loaded amplifiers

The scope of NIF beam control is unprecedented for laser facilities Each of the 192 beamlines has at least 110 optical components and a 500-meter \pm 10 meter path The current design calls for 576 alignment beams, 1200 alignment references, 114 sensor packages, 538 CCD cameras, 9500 stepper motors, 240 photodiodes, 216 calorimeters, and 192 wavefront sensors and deformable mirrors Successful operation of such a system requires a high level of automation Beam controls packages used to accomplish such automation are distributed along each beam line The devices in these packages are linked to front-end processors (FEPs) which are coordinated by a supervisory control system in the NIF Integrated Computer Controls System (ICCS) Within the layered architecture of the ICCS, FEPs perform sequencing, data acquisition and reduction, and device control, and the supervisory control system conducts laser shots, provides status and control interfaces for human operators, processes and atchives laser and target diagnostic output, and coordinates manual device control

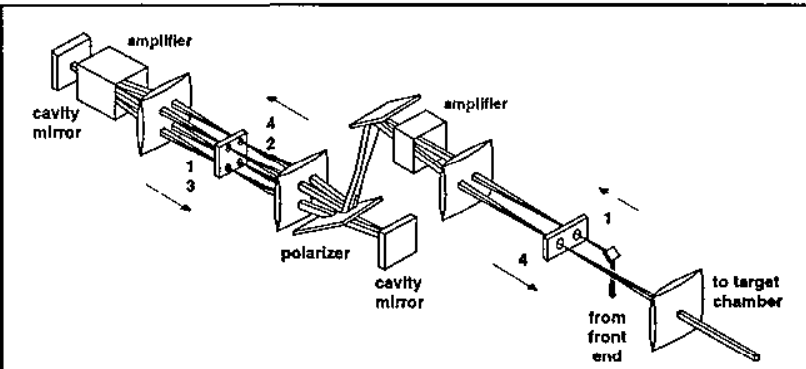

Figure 1 Shot pulses must be steered such that they arrive on target and that, in making multiple passes through the NIF main amplifier, they are not clipped by hardware apertures

This paper summarizes the integrated design approach taken for NIF laser alignment, wavefront correction, and beam diagnostics systems It describes how functions have been automated to ensure reliable and efficient operation, demonstrates how cost and complexity have been controlled, and how performance risk is being reduced by a program of prototype testing

\section{ALIGNMENT}

For a beam to be experimentally useful, it must be precisely steered to designated target chamber aimpoints In order to bound the effective clear aperture of the 
amplifier chain optical elements, the beam must also be precisely aligned throughout the entire laser chain In order to achieve efficient frequency conversion, the main amplifier output must be incident on converter crystals at a specific angle The automatic alignment system accomplishes this alignment by extracting the relative position of beams and references from digitized video images that are distributed throughout the facility on a dedicated network, and using this data to generate crosscoupled, closed-loop control commands These commands null misalignments by operating 31 motors on 14 mounts to control the tip, tilt, and longitudinal positions of 19 optical elements in each of the $192 \mathrm{NIF}$ laser chains

Beam center and orientation are defined in the preamplifier module (PAM) by near field references inserted at the apodizer plane in the beam shaper assembly, and fiber sources identify subsequent aperture centers and orientations Beam pointing is defined primarily by far field references in the NIF spatial filters

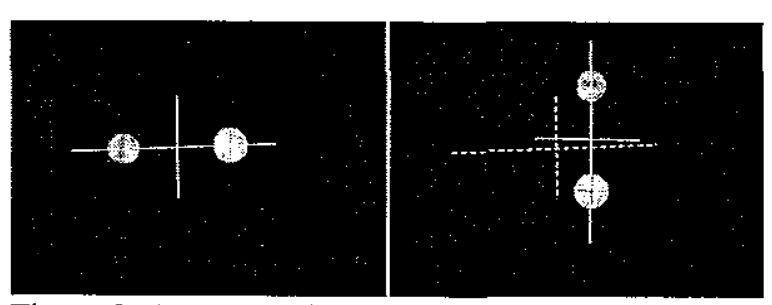

Figure 2 A camera views different relay planes in (a) and (b), recording the location of each plane's reference pair The midpoint of the line joining a reference pair coincides with the center of an aperture The angle between the line joining one reference pair and the line joining a second reference pair corresponds to the rotation the shot pulse will undergo in propagating between the reference pairs

Comparison of two far field images, one of a reference, the other of an alignment laser, provides pointing error signals All but two of the far field references are located inside spatial filters The beam angle relative to the optical axis of a spatial filter can be deduced if the location of the beam focus in the pinhole plane is known The location of the beam focus and pinhole center for a given pass are extracted from pinhole plane images of an alignment beam and reticle Motion of mirrors located outside the spatial filter may be used to null the beam displacement from the center of the far field reference, thus correcting the beam pointing through the shot pinhole for the corresponding pass Figure 2 and Figure 3 contain sample images collected by prototype
FEPs in a scale model of a NIF main amplifier Alignment loops that were simulated included both pointing and centering functions performed using 8-axis, cross-coupled motion of 3 mirrors and 1 polarizer

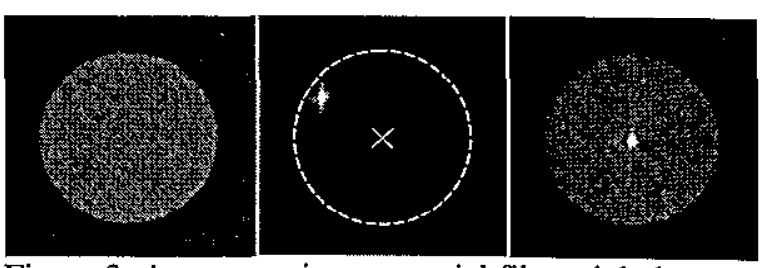

Figure 3 A camera views a spatial filter pinhole plane, recording locations of the reticle and the alignment laser (a) The center of the reticular mark coincides with the center of the pinhole (b) The position of the alignment beam relative to the pinhole center is found by viewing the beam scatter off the reticle (c) The alignment beam is pointed through the pinhole center by changing the angle at which it enters the spatial filter

The $1053 \mathrm{~nm}(1 \omega)$ light from the Main Laser is ftequency upconverted to $351 \mathrm{~nm}(3 \omega)$ before being directed onto targets The final lens in each beam is designed to focus $3 \omega$ light at target chamber center $1 \omega$ light will focus past target chamber center and thus cannot be used for target alignment The irradiance of the alignment laser is too low for appreciable upconversion, therefore it cannot be used to produce a $3 \omega$ alignment beam Fibers are used to inject $3 \omega$ light downstream from the final amplifier, in the $3 \omega$ focal plane of the final spatial filter output lens Introduction of the target alignment source at this location in the chain increases the availability of parallel automatic alignment processes since doing so eliminates the need to wait for amplifier cool down prior to aligning beams to the target $\mathrm{A}$ translatable stage is responsible for precisely positioning an output-coupled fiber on the axis of the pass 4 pinhole in the $3 \omega$ focal plane of the final spatial filter lens The $3 \omega$ light emerges collimated from the spatial filter and follows the beamline through the FOA into the Target Chamber

The Chamber Center Reference System (CCRS), consisting of two high-resolution viewers mounted outside windows at orthogonal Target Chamber ports, provides a stable target chamber coordinate system The position and orientation of the Target Alignment Sensor (TAS) in this coordinate system are sensed and automatically controlled for each shot The TAS provides superimposed views of the target and the $3 \omega$ beams without allowing any beams to preheat the target A fivedegree of freedom positioner is used to place the target in 
the center of the TAS, and the final transport mirror on each beam is controlled to position the beam at the desired position on the target

\section{DIAGNOSTICS}

Laser Diagnostics provide measurements of beam spatial profile, pulse energy, and temporal pulse shape that are required to monitor the health of the laser system and provide feedback to make adjustments for balancing beam characteristics at the target Laser performance is recorded throughout the laser chain so that overall performance degradations may be investigated and localized in a systematic manner While there are significant diagnostic capabilities on each beamline, it will, at times, be necessary to calibrate or expand upon these fundamental diagnostics Therefore, ancillary diagnostics, usable on one or a few beamlines, will be deployed Scheduled inspection of vacuum loaded beamline optics will be carried out between full system shots using video-imaging systems Damaged optics will be replaced well before implosion risk is significant

Several packages, most dedicated, some roving, are used to record laser performance throughout the laser chain The Input Sensor (IS), in addition to providing certain alignment functions, characterizes the first stage of the laser by sampling the output of the regenerative amplifier, the beam shaper, and the multipass amplifier The coatings of three mirrors in the first stage are designed to transmit a small fraction of incident light These leakages are collected by and divided up inside the IS, where the energy is measured and the temporal and spatial pulse shapes are recorded The energy measurement is carried out with an integrating sphere and photodiode followed by a charge integrator and digitizer The temporal pulse shape is measured in a power sensor package external to the IS A CCD camera that images the multipass amplifier output records the spatial pulse shape The imaging resolution is $1 \%$ of the beam dimension and $2 \%$ of the maximum irradiance A sample of the output of the multipass amplifier is sent, via an optical fiber bundle, to the power sensor, where it is time multiplexed with other signals

Each power sensor module temporally multiplexes 12 signals to minimize costs Samples are delivered to the power sensor via fiber optic bundles of specified length Temporal separation is achieved by judiciously choosing fiber lengths, optically introducing delay for signals close in time Each power sensor accepts signals from 4 ISs and 8 OSs, a total of $81 \omega$ signals and $43 \omega$ signals Inside the power sensor, the fiber bundles feed a vacuum photodiode, the signal of which is divided into 4 channels, each with a different sensitivity, that are read out by a transient digitizer The sensor operates with a dynamic range of 50001 , a record length of $22 \mathrm{~ns}$, an accuracy of $2 \%$ over a 2 ns interval, and a rise time of $250 \mathrm{ps}$

The Output Sensor (OS), in addition to providing certain alignment and wavefront correction functions, characterizes the main amplifier and most aspects of the final optics assembly (FOA) A beamsplitter is located immediately downstream from the output lens of the transport spatial filter (TSF) This beamsplitter reflects $01 \%$ of the TSF output back into the TSF The tilt of the sampling surface is set such that the reflected light converges towards a mirror located near the focal plane of the TSF This mirror reflects the sample out of the TSF vacuum vessel and into relay optics which carry it to the OS This pickoff technique illustrated in Figure 4, uses existing large aperture main beamline optics to reduce the beam samples to manageable dimensions, and is used to collect both $1 \omega$ and $3 \omega$ samples In the $1 \omega$ case, the sample is collected off of the aforementioned beamsplitter, in the $3 \omega$ case, the same is collected off of the first, planar surface of the final focus lens

Figure 4 A weakly reflective beamsplitter sends
$01 \%$ of the TSF output back into the TSF The tilt of
the sampling offsets the reflected beam from the
output beam so that a pickoff deployed near focus
can direct the sample into diagnostics

The diagnostic tasks performed by the OS completely characterize the output of the main amplifier, measuring wavefront, and recording spatial and temporal pulse shapes It also records spatial and temporal pulse shapes of the $3 \omega$ that reflects off of the final focus lens Maximum use of multiplexing is utilized to record the performance of so many beams with high accuracy and at a reasonable cost The outputs of two Shack-Hartmann wavefront sensors can be fit onto a single $1 / 2$ " format CCD detector array, so simultaneous measurement of the wavefront of both beams in each pair is possible Energy measurement is accomplished in the relay optics, wherein a beamsplitter the $1 \omega$ beam path is used to direct a sample into a photodiode assembly like that in the IS Each beamline has a dedicated photodiode, so pulse energy is measured for every beam every shot As mentioned 
above, samples of $1 \omega$ and $3 \omega$ light are sent to the power sensor The power of one beam from every pair of beams is measured for each shot This is the only OS diagnostic for which simultaneous use is not possible CCD cameras image planes in the Target Bay by collecting light reflecting off of the final focus lens Two images are fit onto both the $1 \omega$ and the $3 \omega$ CCD cameras, so every beam is imaged every shot

The final fixed diagnostic package is the $3 \omega$ calorimeter The final optics diagnostics uses a diffractive splitter to obtain a sample for the $3 \omega$ calorimeter By measuring the $3 \omega$ energy, this calorimeter calibrates the $3 \omega$ beam power for each shot, inasmuch as the integrated $3 \omega$ temporal pulse shape is directly proportional to the total $3 \omega$ pulse energy

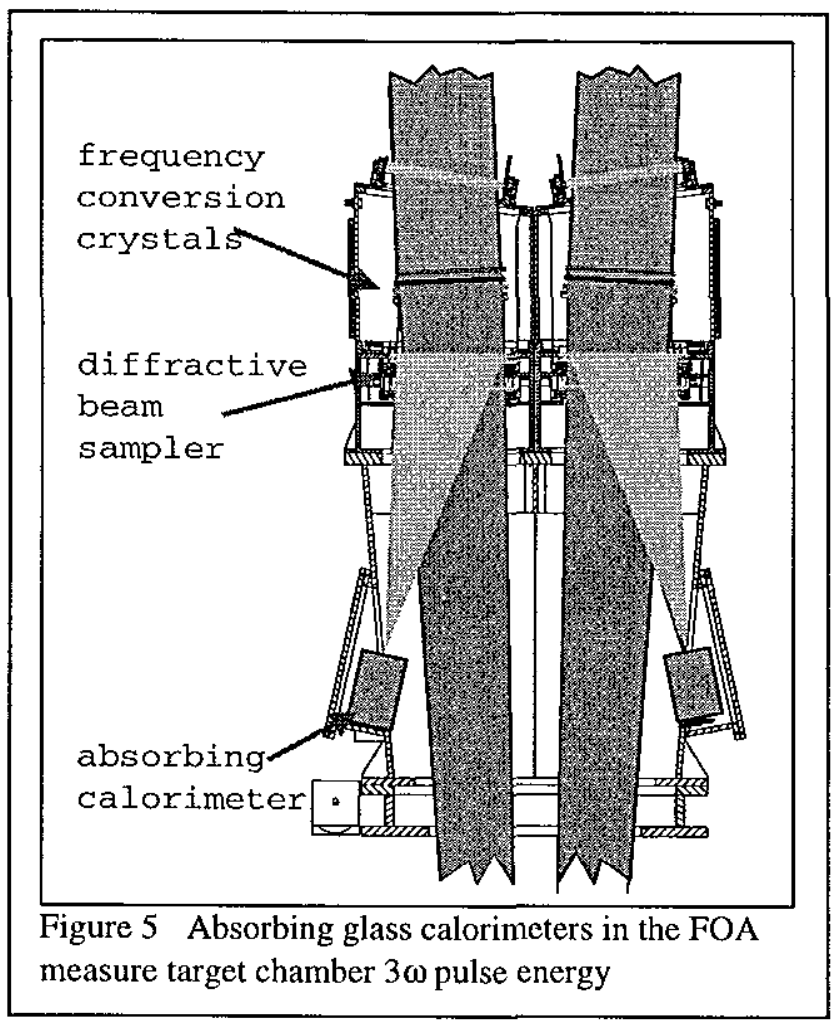

One online inspection system is located in each switchyard, and one more at target chamber center These systems have access to each beamline through a set of translatable mirrors The requirement for main-beamline optics is to detect flaws $\geq 5 \mathrm{~mm}$, which is $1 / 4$ of the critical flaw size The system uses dark field imaging Damage spots tend to scatter, but not completely obstruct light being transmitted by an optic light In conventional images of an optic operating in transmission, damage sites would appear as slight nonuniformities in a bright object In dark field imaging, undisturbed light is intercepted by an axial obscuration at a focal plane in the imaging optics while light diffracted by damage spots forms images as in a conventional system In such images, damage spots appear as bright images on a dark background

Pulse synchronization is established using a target chambet diagnostic A module is inserted to target chamber center and oriented so as to capture the output of the four beams of a single FOA The preamplifier is fired to produce a measurable amount of $1 \omega$ at TCC The $1 \omega$ of each of the four beams from an FOA is captured and directed into its own fiber bundle The fiber bundles carry the optical signals to a streak camera where their relative times of arrival are compared to an electronic timing reference fiducial

Full aperture calorimeters and a suite of precision diagnostics complete list of diagnostics Each switchyard has a roving calorimeter assembly, which consists of an array of eight calorimeters that can move to intercept from 1 to 8 of the beams in a bundle These calorimeters are used to calibrate the main amplifier photodiodes Portable calorimeters may also be attached to the IS, measuring the entire preamplifier module output for the purpose of calibrating the IS photodiodes

The precision diagnostic station is a shared diagnostic that measures laser output performance one beam at a time using more extensive instrumentation than that found in the OSs The precision diagnostics will measure frequency conversion characteristics of the selected beam using a separately selected module containing the frequency conversion crystals and final focus lens The precision diagnostics provide the only capability for simultaneously measuring high-power $3 \omega$ beam properties the full $40-\mathrm{cm}$ aperture and in a plane equivalent to the target chamber focus In cases where this station measures parameters also measured by the $\mathrm{OS}$, it does so with higher degrees of accuracy, precision, and resolution

\section{WAVEFRONT CORRECTION}

During preparations for a pulsed shot, the wavefront control system monitors the wavefront of each alignment laser at the main amplifier output and automatically compensates for measured aberrations using a fullaperture deformable mirror There are static aberrations induced by optical fabrication and mounting errors, and dynamic aberrations induced by flashlamp light at shot time, by residual heat in amplifiers after a shot, and from gas density gradients in the propagation path In the last few minutes before a shot, the controlled wavefront is 
biased to include precorrections for the estimated dynamic aberrations caused by firing the flashlamppumped amplifiers One second before a shot, closedloop operation is interrupted, and the wavefront sensor is configured to measure the pulsed shot wavefront The measured pulsed shot wavefront error provides additional information for setting precorrection wavefronts prior to the next shot

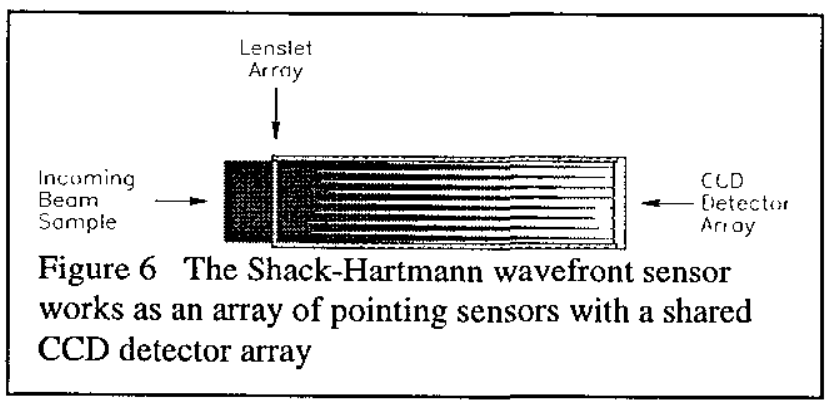

The three main components of the wavefront control system are a Shack-Hartmann wavefront sensor, a deformable mirror, and a computer controller The Shack-Hartmann sensor, illustrated in Figure 6, includes a 2 dimensional array of lenslets and a CCD video camera located in the back focal plane of the lenslets The output sensor delivers a demagnified image of the main amplifier output to the lenslet array The plane that is imaged is conjugate to the plane of the deformable mirror Each lenslet collects light from a specific part of the beam and focuses it on the CCD The position of the lenslet's focus on the CCD give a measure of the pointing of the small portion of the total beam that is incident on that particular lenslet Directional data from the 77 hexagonally packed lenslets of the NIF sensor is processed to determine the output wavefront with an accuracy of $\leq 01$ wave and a spatial resolution of $45 \mathrm{~cm}$ in the $40-\mathrm{cm}$ beamline aperture

The NIF design includes 192 large-aperture deformable mirrors for wavefront control in the main laser cavity The required optical clear aperture is approximately $400 \mathrm{~mm} \times 400 \mathrm{~mm}$ The mirror shape will be determined by the displacements of 39 replaceable actuators spaced $80 \mathrm{~mm}$ apart Prototype efforts are under way, three deformable mirrors, two commercially produced and one built at Lawrence Livermore National Laboratory, will soon be ready for testing The two main specifications to be tested are actuator lifetime and residual wavefront error The mirrors serve as an end mirror for the main laser amplifier cavity In this configuration, mechanisms located between the mirror substrate and reaction plate, namely the mirror actuators, are exposed to high fluence flashlamp light As part of design qualification, the prototypes must survive repeated exposure to $10 \mathrm{~J} / \mathrm{cm}^{2}$ pulses of light in the NIF flashlamp test facility The residual wavefront error will be measured in a large aperture interferometer, wherein a flat wavefront will be incident upon the prototypes The wavefront controller will be activated and the reflected wavefront measured A design will be qualified if its prototype imparts less than 0025 waves of rms wavefront error

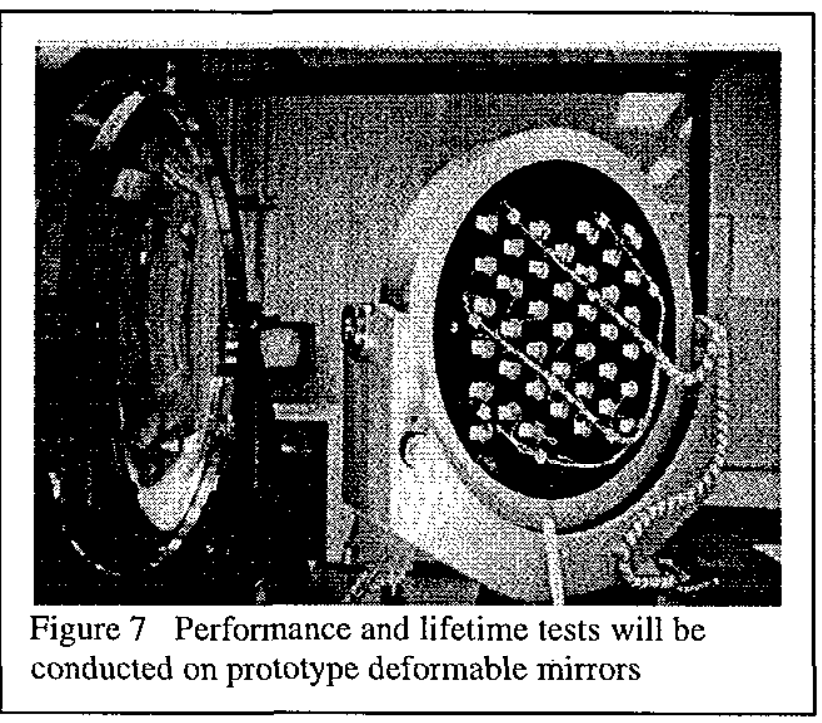

Each wavefront controller comprises computer hardware and software to periodically calibrate the associated wavefront sensors and deformable mirrors, operate the automatic wavefront correction loops during preparations for a shot, and capture pulsed wavefront measurement data during a shot Since the ShackHartmann sensor data is in video format, the controller incorporates image processing capabilities appropriate for recognizing and tracking the position of the 77 focused spots from each sensor image The image processing code attains maximum accuracy by automatic adjustment of software parameters for grayscale and brightness The controller also measures and applies the influence matrix for the deformable mirror actuators and the amplifier precorrection file in accordance with the mirror control algorithm When operating in closed-loop, the controller is intended to maintain a bandwidth of approximately $1 \mathrm{~Hz}$ on each beam

\section{$\mathrm{V}$ ACKNOWLEDGEMENTS}

This work was performed under the auspices of the United States Department of Energy by Lawrence Livermore National Laboratory under contract number W-7405-Eng-48 


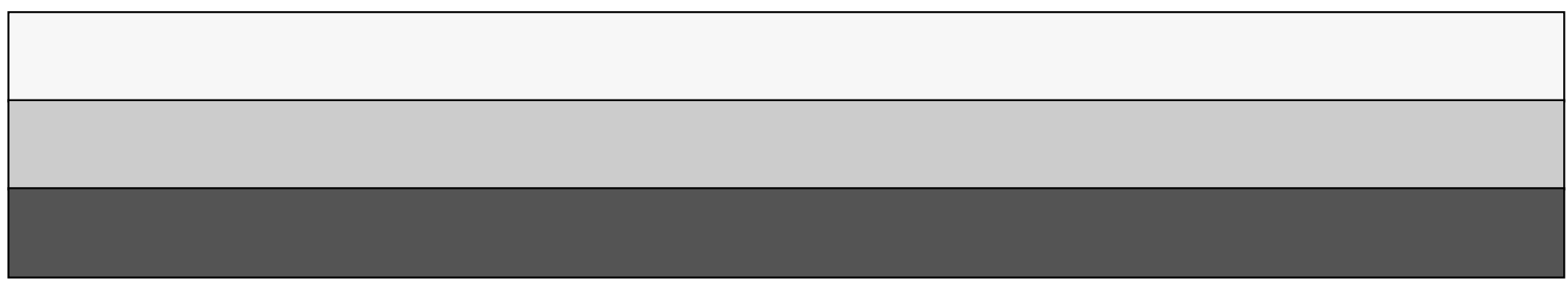

